\title{
Bacteriological Analysis Including Antimicrobial Susceptibility Pattern of Blood Stream Infections in Tertiary Care Hospital
}

\author{
Iqbal M Desai', Hardik Kamlesh Bhavsar", Sachin M Darji² and Jitendrakumar S. Parmar ${ }^{3}$ \\ ${ }^{1}$ Department of Microbiology, Gujarat Research and Medical Institute. Rajasthan Hospitals. Ahmedabad. India \\ ${ }^{2}$ Department of Microbiology, GCS Medical college, Hospital and Research centre. Ahmedabad. India \\ ${ }^{3}$ Dept of Pathology, Gujarat Research and Medical Institute. Rajasthan Hospital, Ahmedabad. India
}

\begin{abstract}
Background: Blood stream infections cause significant morbidity and mortality worldwide. Illness associated with blood stream infection ranges from self-limiting infections to life- threatening sepsis that require rapid and aggressive antimicrobial treatment. Rational and correct use of antibiotic requires understanding of common pathogens and their drug resistance pattern in the community as well as hospital.
\end{abstract}

Methods: A retrospective study was conducted on the microbial profile of isolates of blood culture and their antimicrobial sensitivity pattern in a tertiary care hospital. Blood culture samples received from January to December 2015 in Microbiology department of the laboratory were enrolled in the study.

Result: Positivity of the blood culture was found to be $16.66 \%$ (312/1872). Among the isolates, Gram negative organisms were $66.76 \%$ (203/300) while gram positive organisms were 32.33\% (97/300) including $45(15 \%)$ fungal isolates which were of various candida species. Klebsiella species was the most commonly isolated organism (27.3\%) followed by E.coli (18.6\%) and Acinetobacter species (8.3\%). Staphylococcus aureus was isolated in $6 \%$ cases. Colisitin was found to be showing $100 \%$ sensitivity for Klebsiella, E.coli, Acinetobacter and Pseudomonas. Vancomycin was found to be sensitive for all Staphylococci isolated from blood. Ampicillin failed to show significant sensitivity against any of the above mentioned organisms.

Conclusion: Specific antibiotic usage strategies to be prepared and implemented in the form of antibiotic usage policy like antibiotic restriction, combination therapy and usage according to the standard antibiotic susceptibility testing are needed for each tertiary care hospital to prevent emergence and spread of drug resistance.

Keywords: Antibiotics, Blood Stream Infection, Bacteria, Antimicrobial Susceptibility.

\section{Introduction}

Blood stream infections cause significant morbidity and mortality worldwide and are among the most common healthcare associated infections. Microorganisms present in circulating blood whether continuously or intermittently are a threat to every organ in the body. Approximately, 200,000 cases of bacteremia and fungemia occur annually with mortality rates ranging from $20-50 \% .{ }^{[1]}$

Neonatal sepsis is a significant cause of neonatal morbidity and mortality in the newborn, particularly in preterm and low birth weight infants. According to World Health Organization (WHO) estimates, neonatal sepsis remains the major cause out of five million neonatal deaths per year. ${ }^{[2]}$

Since early 1950s, there has been a striking increase in incidence of bacteraemia caused by members of enterobacteriaceae and other gram negative bacteria. Escherichia coli which was reported to be common in the past is being replaced by other multidrug resistant bacteria like Klebsiella, Enterobacter, Salmonella, Pseudomonas, Acinetobacter, etc.

Illness associated with blood stream infections range from self- limiting infections to life-threatening sepsis that requires rapid and aggressive anti-microbial treatment. The incidence of blood stream infections in patients has been reported to correlate with the increasing use of central venous catheters, patient illness (e.g., oncology, burn, trauma and high risk nursery), and other predisposing factors including intensive care unit (ICU) stay, lapses in hand washing and non adherence to infection control practices of medical staff. Respiratory, genitourinary, and intra abdominal foci are often identifiable foci of blood stream infections. ${ }^{[3]}$

Nowadays, bacterial drug resistance is an important problem and due to wide variations in bacterial drug resistance, results of studies and reports in one region or in one period of time are not necessarily true for other regions or periods of time. They are related with a series of 
social, environmental and technological changes. ${ }^{[4],{ }^{[5]} \text { The }}$ isolated bacteria are numerous and their associated diseases need urgent and invasive management with antimicrobial agents. Rational and correct use of these agents requires understanding of common pathogens and drug resistance pattern in the region. [6] The varying microbiological pattern of septicemia warrants the need for an ongoing review of the causative organisms and their antimicrobial susceptibility pattern.

A study was conducted to identify the bacteriological profile and their antibiotic susceptibility patterns by analyzing the data on the blood culture isolates of a tertiary care hospital.

\section{Materials and Methods}

In this retrospective study, 1872 blood samples from patients clinically suspected of having bacteremia, were collected during the period of January to December 2015 in a tertiary care hospital. Processing of the samples was done at the Microbiology Department of the same hospital.

8-10 $\mathrm{ml}$ and 4-6 $\mathrm{ml}$ blood was collected using aseptic precautions by the trained staff nurses from adult and pediatric patients, respectively. Blood was transferred into respective BACTEC aerobic and BACTEC Ped plus blood culture bottles, at the blood collection site. Inoculated blood culture bottles were transferred to Microbiology laboratory immediately where they were loaded into BACTEC machine according to operational guideline for BACTEC. Positive bottles were identified by the machine and were then inoculated onto 5\% sheep blood agar and MacConkey agar. Organism was identified using Gram stain technique, colony morphology and various biochemical tests. ${ }^{[7]}$ Antibiotic susceptibility testing of the isolated organisms was performed using Kirby Bauer's disc diffusion method on Mueller Hinton agar plates. ${ }^{[8]}$ The antibiotic discs that were used to identify the susceptibility pattern of the bacterial pathogens along with their concentrations are: Ampicillin (10 mcg), Amikacin (30 mcg), Cefotaxime (30 mcg), Ceftazidime (70 mcg), Cefepime (30 mcg), Cefoperazone + sulbactum $(70 \mathrm{mcg})$, Levofloxacin (5 $\mathrm{mcg}$ ), Cotrimoxazole (trimethoprim /sulphamethoxazole $1.25 / 23.75 \mathrm{mcg})$, Erythromycin (10 mcg), Gentamicin (10 $\mathrm{mcg}$ ), Imipenem (10 mcg), Linezolid (30 mcg), Piperacillin + tazobactum (100/10 mcg), Colistin (10 mcg) and Vancomycin $(30 \mathrm{mcg})$. The data obtained were tabulated and analyzed to identify the common causative pathogens of blood stream infections and the antibiotics to which the identified organisms were sensitive and resistant. The results obtained were expressed by descriptive statistics.

\section{Result}

A total of 1872 blood culture samples were sent to Microbiology lab during the period of January to
December 2014, of which 312(16.66\%) were identified as culture positive samples. The gender distribution of positive samples was found to be $278(89.1 \%)$ males and $34(10.89 \%)$ females. The positive samples belonged to $245(78.52 \%)$ adults (age range $>18$ years) comprising of $221(90.20 \%)$ male and $24(9.79 \%)$ female patients, $15(4.80 \%)$ adolescents (age range 13-18years) which included $14(93.33 \%)$ males and $1(6.66 \%)$ female patients. $37(11.85 \%)$ children (age range 1-12 years) which included $31(83.78 \%)$ males and $6(16.21 \%)$ female patients. $15(4.80 \%)$ infants (age range $<1$ year) which included 11 $(73.33 \%)$ males and $4(26.66 \%)$ female patients.

Out of 312 positive cultures, $300(96.15 \%)$ showed bacterial growth. Out of those 300 isolates, 97 (32.33\% ) were grampositive which included candida which is fungus and 203 $(67.66 \%)$ were gram-negative. The most commonly isolated gram-positive bacteria were Staphylococcus aureus in $22(42.3 \%)$, Enterococci in $12(23.07 \%)$, Streptococcus in $3(5.76 \%)$ out of total gram positive bacterial isolates i.e.52. The most prevalent gram-negative bacteria found in positive cultures were Klebsiella pneumoniae in 82 (40.39\%) cultures, followed by Escherichia coli in 56 (27.58\%), Salmonella typhi 25 (12.31\%), Pseudomonas aeruginosa in 20(9.85\%), Acinetobacter baumannii in 12 (4\%) culture specimens out of all isolated gram negative bacteria. [Figure 1].

The results of antibiotic drug sensitivity of gram positive bacteria showed that Staphylococcus aureus was highly sensitive to Vancomycin (100\%) and Linezolid (100\%) followed by Levofloxacin (68.18\%). Enterococci also were highly sensitive to Linezolid (100\%) and Vancomycin (75\%) followed by Levofloxacin (41.6\%). Streptococci were found to be highly sensitive against Ampicillin $(100 \%)$. So of all the antibiotics, Vancomycin and Linezolid were found to be most active against Staphylococcus aureus and Enterococci. [Table 2].

The results of antibiotic drug sensitivity of gram negative bacteria showed the following results. Klebsiella, E. coli and Pseudomonas showed 100\% sensitivity towards Colistin. Acinetobacter also showed maximum sensitivity towards Colistin (83.33\%). Klebsiella showed maximum sensitivity to Imipenem (79.26\%) after Colistin; followed by Piperacillin+Tazobactum (43.90\%), Cefoparazone+Sulbactum (36.02\%) and Amikacin (30.48\%). All isolated Klebsiellae were resistant to Cefotaxime. E. coli also showed maximum sensitivity towards Imipenem (92.85\%) after Colistin, followed by Amikacin (78.57\%), Piperacillin+Tazobactum (64.28\%), Cefoparazone+Sulbactum (60.71\%).E coli 
showed least sensitivity towards Cefotaxime (8.92\%). Levofloxacin however, was sensitive in $35.71 \%$ isolates. Acinetobacter showed maximum sensitivity towards Colistin (83.33\%) and Imipenem (66.66\%) followed by Cefoparazone+Sulbactum and Amikacin (33.33\% each). Piperacillin+Tazobactum showed sensitivity of $25 \%$ and Levofloxacin showed $16.66 \%$ sensitivity. Cefotaxime or Cefepime did not show any sensitivity against Acinetobacter isolates. Pseudomonas aeruginosa showed maximum sensitivity towards Imipenem (80\%) after Colistin(100\%), followed by Piperacillin + Tazobactum and Cefoparazone + Sulbactum $(75 \%$ each). Amikacin showed $60 \%$ sensitivity, same as Levofloxacin. Cefepime showed $20 \%$ sensitivity while Cefoparazone could show only $10 \%$ sensitivity. Salmonella typhi showed 100\% sensitivity against cephalosporins like Cefotaxime. Levofloxacin however was either resistant or intermediately sensitive for $60 \%$ Salmonella isolates.[ Table 1]

Table 1: Antimicrobial susceptibility for various gram negative bacterial isolates in percentage out of total number of isolates of the organism.

\begin{tabular}{|c|c|c|c|c|c|c|c|c|c|c|c|}
\hline & $\begin{array}{l}\frac{E}{\Phi} \\
\frac{0}{0} \\
\text { 을 }\end{array}$ & 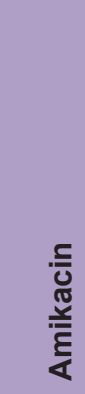 & 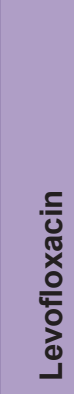 & 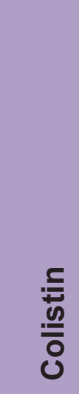 & $\frac{.}{\frac{.}{\bar{O}}}$ & 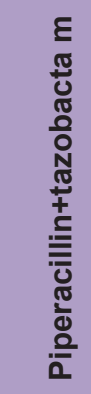 & 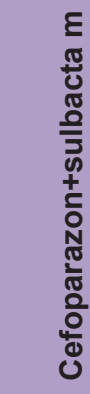 & $\begin{array}{l}\frac{\varepsilon}{x} \\
\frac{\pi}{0} \\
\frac{0}{0} \\
\frac{0}{0} \\
0\end{array}$ & 这 & 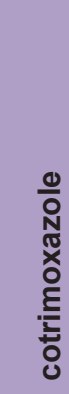 & 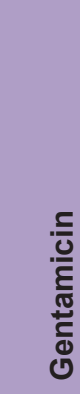 \\
\hline Klebsiella & 65 & 25 & 20 & 82 & 0 & 36 & 30 & 0 & 0 & 15 & 20 \\
\hline E.coli & 52 & 44 & 20 & 56 & 0 & 36 & 34 & 5 & 5 & 15 & 35 \\
\hline Acinetobacter & 8 & 4 & 2 & 12 & 0 & 2 & 4 & 0 & 0 & 1 & 1 \\
\hline Pseudomonas aeruginosa & 16 & 12 & 12 & 20 & 0 & 15 & 15 & 2 & 4 & 0 & 10 \\
\hline Salmonella typhi & 25 & 0 & 15 & 25 & & 25 & 25 & 25 & 25 & 25 & 0 \\
\hline Salmonella paratyphi A & 4 & 0 & 3 & 4 & 4 & 4 & 4 & 4 & 4 & 4 & 0 \\
\hline
\end{tabular}

Table 2: Antimicrobial susceptibility of gram positive bacterial isolates in percentage out of total number of the organism isolated.

\begin{tabular}{|c|c|c|c|c|c|c|c|c|c|c|c|c|}
\hline & 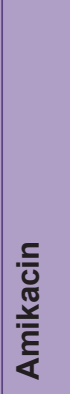 & 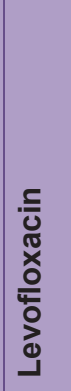 & $\frac{\text { 亭 }}{\frac{0}{\frac{0}{2}}}$ & 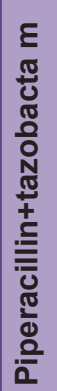 & 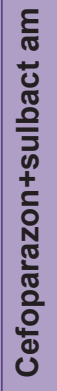 & 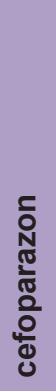 & 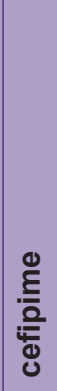 & 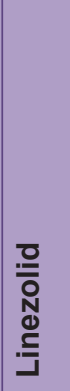 & 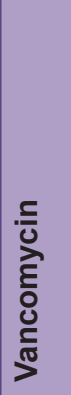 & 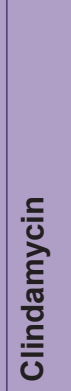 & 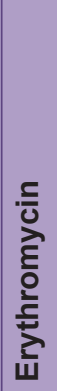 & 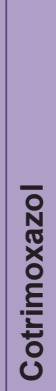 \\
\hline Staphyl ococcus aureus & 15 & 15 & 5 & 5 & 5 & 5 & 5 & 22 & 22 & 12 & 8 & 2 \\
\hline Staphyl ococcus CONS & 4 & 10 & 4 & 0 & 0 & 0 & 0 & 15 & 15 & 0 & 0 & 0 \\
\hline Enteroc occi & 0 & 5 & 5 & 5 & 0 & 0 & 0 & 12 & 9 & 0 & 0 & 0 \\
\hline Streptoc occus pyogenu s & 3 & 3 & 3 & 3 & 3 & 3 & 3 & 3 & 3 & 3 & 3 & 3 \\
\hline
\end{tabular}




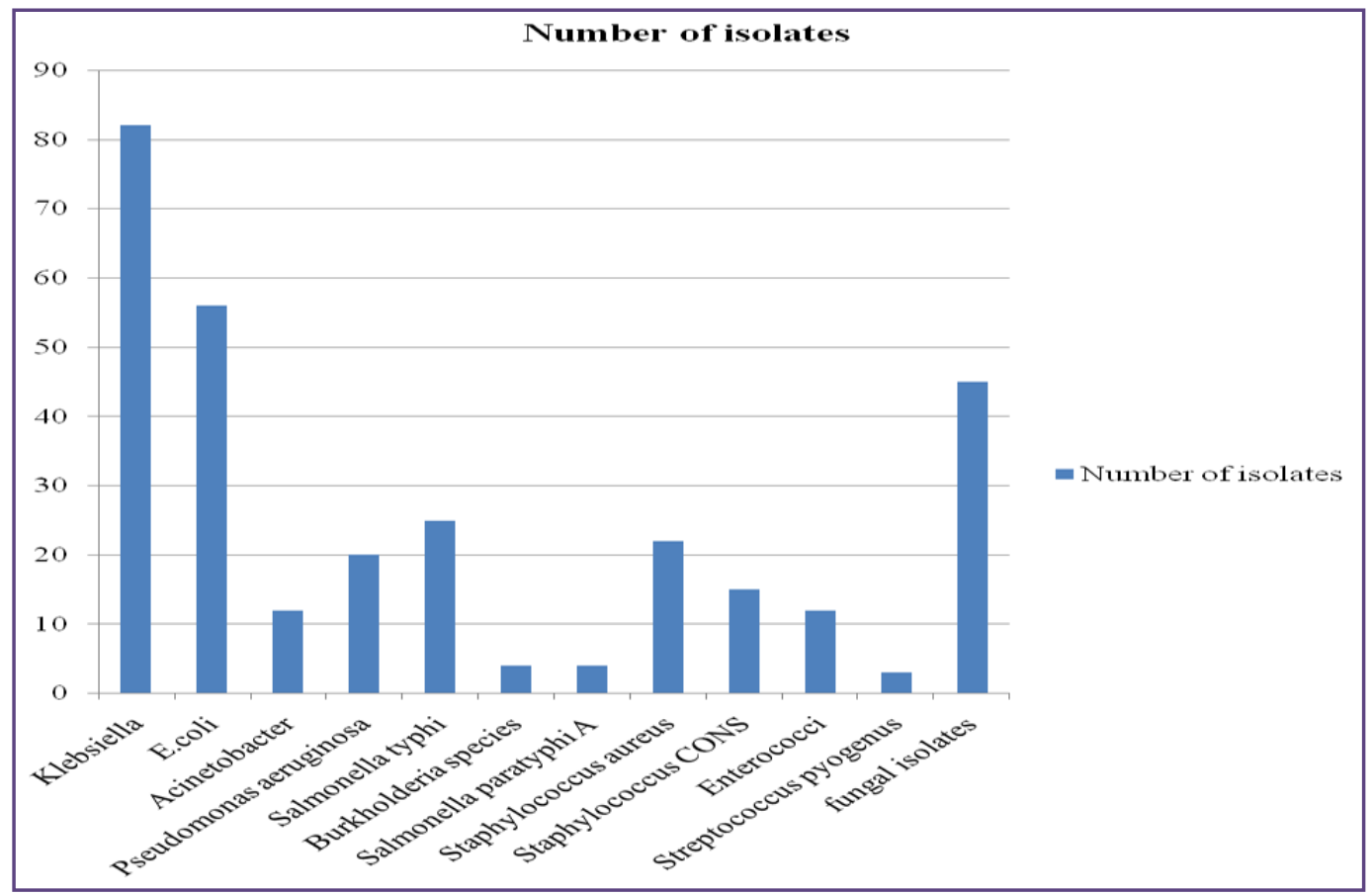

Fig. 1: Number of various isolates from positive blood cultures.

\section{Discussion}

Bacteriological profile and the antimicrobial susceptibility are constantly evolving. Study of bacteriological profile with antibiotic susceptibility pattern plays an important role in effective management of bacteremia cases. Many studies have been undertaken to determine the organisms responsible for blood stream infections all over the world. Results have varied in different centers and different parts of the world. Many factors e.g. socioeconomic, geographic, use of ventilators, and administration of different antibiotics etc., play a very important role in explaining these differences. The results of the retrospective study conducted in our tertiary care hospital demonstrated that $312(16.66 \%)$ out of 1872 total blood samples screened were positive for the presence of micro-organisms of which $255(85 \%)$ were bacteria and $45(15 \%)$ were fungi. A study conducted by Mehta et al also reported similar frequency of positive blood culture as $16.4 \%$. ${ }^{[9]}$

In contrast to above reports, the study done by Khanal et al ${ }^{[8]}$ and Sharma ${ }^{[10]}$ reported high frequency of positive blood cultures accounting for $44 \%, 33.9 \%$ and $20.2 \%$ respectively. Whereas studies by Anbumani et $\mathrm{al}^{[11]}$ and Arora $^{[12]}$ reported lower frequency of positive blood cultures accounting for $7.89 \%$ and $9.94 \%$,respectively.

The variation in blood culture positivity is related to different factors such as the number and amount of blood cultures taken for screen as reported by Lee et al. ${ }^{[13]}$ They believed that for achieving a detection rate of $>99 \%$ as many as four blood cultures may be needed. Similar comment was made by other investigators that more than three blood cultures are needed for $99 \%$ test sensitivity. In India, variation might be due to the fact that most of the patients are given antibiotics before they come to the tertiary care hospital and other reason is that in most of the cases self medication is very common as the antimicrobials are available over the counter.

In most of the studies, gram-negative bacilli have taken over the gram-positive organisms, especially in hospital settings. Mehta et $\mathrm{al}^{12}$ have reported the incidence of $80.96 \%$ culture positivity for gram-negative and $18 \%$ for gram-positive organisms which is similar to our findings. Our study revealed that gram-negative bacteria were predominant $(66.76 \%)$. Klebsiellae were reported to be the most common gram-negative organisms isolated from blood stream infections in many studies like present study. In the present study, we also observed a high frequency of E. coli. (18.66\%). The high occurrence of non-lactose fermenters especially Pseudomonas spp.(6.66\%) and Acinetobacter spp (4\%). is of concern. Both of these bacteria are associated with a high degree of resistance to antibiotics. Another important finding of this study was high rate of Salmonella isolation $(8.33 \%)$.

The incidence of gram-positive organisms is $32.33 \%$, including Candida, in our study.Gram positive bacterial isolates were $17.33 \%$. Among the total gram positive bacterial isolates $(n=52)$, the most common was 
Staphylococcus aureus (42.3\%), followed by Enterococcus faecalis (23.07\%) Staphylococcus seems to be emerging as the dominant organism in blood stream infections with $45 \%$ MRSA isolates. Here the percentage were calculated out of total gram positive bacteria isolated. Similar trend has been reported in the data from the west over the last two decades. Nosocomial infection due to Staphylococcus aureus constitutes a major part of the total annual nosocomial infections. ${ }^{[14]}$

Enterococcus spp. also, was observed as an important pathogen in our study $(23.07 \% ; n=52)$. They form part of the normal gastrointestinal and female genitourinary tract flora. Over the past decade, Vancomycin resistant enterococci (VRE) have emerged as a leading cause of nosocomial infections due to its spread by direct patient to patient contact and by direct transmission via hospital personnel, environmental surfaces and hospital equipment. In our study, $25 \%$ of Enterococci were VRE. A study by CDC also reported high prevalence of VRE (29\%), similar to our study. ${ }^{[15]}$

In the present study, Candida isolates were seen in 25 $(15 \%)$ cases. This epidemiologic trend has also been observed in many studies in different parts of the world. ${ }^{[16]}$. According to the surveillance data from US Center for Disease Control and Prevention (CDC), Candida accounts for $12 \%$ of all hospital acquired Blood stream infections. ${ }^{[17]}$

Most of the gram negative bacilli in the study were multi drug resistant. The most common resistance was seen to Ampicillin in all isolated gram negative bacteria. Other studies have also reported multidrug resistance for their isolated gram negatives. Colistin, Amikacin, Imipenem and piperacillin + tazobactum were found to be most effective antibiotics for all gram negative bacterial isolates including non- fermenters. Salmonella typhi was found to be highly sensitive to Ceftriaxone. Among the grampositive organisms, high resistance was noted against Ampicillin. An increased ampicillin resistance of $64 \%$, $87 \%$ was also reported by Guha et al ${ }^{[18]}$ and Karki et al ${ }^{[19]}$,respectively in their studies. Resistance to third and fourth generation cephalosporins (cefotaxime, cefixime and cefepime) was also observed with Staphylococcus aureus and Enterococcus faecalis in the present study. This could be due to the abundant use of these drugs; especially third generation cephalosporin's in hospitals, as reported by Nathisuwan et al. ${ }^{[20]}$ The study also showed that Staphyloccocus aureus was found to be highly sensitive to vancomycin \& linezolid.

\section{Conclusion}

Overall, present results indicate that Colistin and Imipenem are highly active against gram negative and Vancomycin and Linezolid are highly active against gram positive organisms causing blood stream infections. However, it should not be expected that this activity continues for a long time. Therefore it is advisable to continuously evaluate the sensitivity-resistance pattern of isolates in each region so as to make a rational use of antibiotics. The present study provided much needed information on the prevalence of bacterial pathogens in blood stream infections and their antibiotic sensitivity patterns. The study identified both gram positive and gram negative bacteria were responsible for blood stream infections and most of them were multi drug resistant. The main forces driving the increase in antimicrobial resistant bacteria are poor infection control practices and inappropriate use of antibiotics. Specific antibiotic utilization strategies like antibiotic restriction, combination therapy and antibiotic recycling may help to decrease or prevent the emergence of resistance and antibiotic usage according to the standard antimicrobial susceptibility testing may reduce the incidence of blood stream infections.

\section{Acknowledgements}

We would like to acknowledge that all the samples were collected and processed in "Gujarat Research and Medical Institute, Rajasthan Hospital, Ahmedabad." We also want to thank the institute for allowing us to conduct the study.

\section{Reference}

1. Beekman SE, Chapin KC. Epidemiology and outcome of nosocomial and community on set blood stream infection. $\mathrm{J}$ clin microbiol 2003; 41: 3655-60

2. WHO. Perinatal Mortality. Report No: WHO/FRH/ MSM/967. Geneva: WHO, 1996.

3. Karlowsky JA, Jones ME, Draghi DCVolturo GA. Prevalence and antimicrobial susceptibilities of bacteria isolated from blood cultures of hospitalized patients in the United States in 2002. Annals of clinical microbiology and antimicrobials 2004;3:7.

4. Reingold AL. Antibiotic resistance patterns of bacterial isolates from blood in San Francisco country, California, 1996- 1999. Emerg Infect Dis 2007; 8: 195-201.

5. Cohen ML. Epidemiological factors influencing the emergence of antimicrobial resistance. Liba Found Symp 1997; 207: 223 - 231.

6. Graham C. Bacteremia and antibiotic resistance of its pathogens reported in England and wales between 1990 and 1998: Trend analysis. Br Med J 2000; 320: 213 - 216.

7. Cruickshank K, Duguid JP, Marmion BP. Test for sensitivity to antimicrobial agents. In: Medical Microbiology. Churchill Livingstone, 1980; 190-209.

8. Khanal B, Harish BN, Sethuraman KR, Srinivasan S. Infective endo carditis: Report of prospective study in an Indian Hospital. Trop Doct 2002; 32:83-85. 
9. Mehta MP, Dutta, V Gupta. Antimicrobial susceptibility pattern of blood isolates from a teaching hospital in North India. Jpn J Infect Dis 2005;58:174-176.

10. Sharma PP, Halder D, Dutta AK. Bacteriological profile of neonatal septicemia. Ind Pediatr 1987;24:1011-1017.

11. Anbumani N, Kalyani J, Mallika M. Original research distribution and antimicrobial susceptibility of bacteria isolated from blood cultures of hospitalized patients in a tertiary care hospital. Indian Journal for the practicing doctor 2008;5(2). Available at: http;//www.indmedica.com/journals. php?journalid +3 \&issueid=1658\&action=article (1of 9$)$.

12. Arora U, Devi P. Bacterial profile of blood stream infections and antibiotic resistance pattern of isolates. J K Sci 2007; 9:186- 190

13. Lee A, Mirrett S, Reller LB, Weinstein MB. Detection of blood stream infections in adults: How many blood cultures are needed? J Clin Microbiol 2007; 45:3546-48.

14. Roy, I. ,A.Jain,M.Kumar and S.K.Agarwal. Bacteriology of neonatal septicemia in a tertiary care hospital of Northern India. Indian J.Med.Microbiol 2002;20:156-159.
15. Centers for Disease Control and Prevention National Nosocomial Infection Surveillance (NNIS) System Report, data summary from January 1992 through June 2004, issued October 2004. Am J Infect Control. 2004;32:470-85.

16. Bassetti M, Righi E, Costa A, et al. Epidemiologic trends in nosocomial candidemia in intensive care, BMC infect dis. 2006;6:21

17. Hidron $\mathrm{Al}$, Edwards JR, Patel J et al. Antimicrobial-resistant pathogens associated with healthcare-associated infections: annual summary of data reported to the National Healthcare Safety Network at the Centre for Disease Control and Prevention 2006-2007. Infect. Control Hosp Epidemiol. 2008;29:996-1011

18. Guha DK, Jaspal D, Das KMS, Guha RA, Khatri RL, Srikumar R. Outcome of neonatal septicemia:A clinical and bacteriological profile. Indian Pediatr 1978;15:423-27.

19. Bassetti M, Righi E, Costa A, et al. Epidemiologic trends in nosocomial candidemia in intensive care, BMC infect dis. 2006;6:21

20. Nathisuwan S, Burgess DS, Lewis II JS. Extended spectrum - lactamases: Epidemiology, Detection and treatment. Pharmacotherapy 2001;21:920-928.

*Corresponding author:

Dr Hardik K Bhavsar, Department of Microbiology, GCS Medical College, Opp. D.R.M office, Naroda road, Ahmedabad, Gujarat, India

Phone: +91 9099084835

Email: bhavsar.dr.hardik@gmail.com

Date of Submission : 13.12.2016

Date of Acceptance : 03.05.2017

Financial or other Competing Interests: None.

Date of Publication : 05.07.2017 